\title{
Social Comparison based Relaying in Device-to-Device Networks
}

\author{
Young Jin Chun*, Gualtiero B. Colombo ${ }^{\dagger}$, Simon L. Cotton*, William G. Scanlon*, Roger M. Whitaker ${ }^{\dagger}$, \\ and Stuart M. Allen ${ }^{\dagger}$ \\ * Institute of Electronics, Communications and Information Technology (ECIT) \\ Queens University Belfast, Belfast, United Kingdom \\ $\dagger$ Computer Science \& Informatics, Cardiff University, Cardiff, United Kingdom \\ Email: $\{$ y.chun, simon.cotton,w.scanlon\}@qub.ac.uk, \{ColomboG, WhitakerRM, AllenSM $\} @$ cardiff.ac.uk
}

\begin{abstract}
Device-to-device (D2D) communications are recognized as a key component of future wireless networks which will help to improve spectral efficiency and network densification simultaneously. In order to guarantee a quality of service (QoS) to the cellular links, the transmit power of the D2D nodes needs to be restricted, which has lead to a poor link quality over D2D transmission. One viable option to improve the D2D link quality is incorporating cooperative relays into D2D networks. However most of the existing published work in relay assisted D2D networks has assumed that relay nodes cooperate spontaneously. This cannot always be guaranteed and we take this into account by considering a fundamental model on which donation-based cooperation depends. In particular we model relay cooperation as a donation game based on social comparison and characterize cooperation probability in an evolutionary context. When applying this model we evaluate the outage and capacity of relay assisted D2D network using a stochastic geometric framework.
\end{abstract}

Index Terms-Cooperative relaying, device-to-device network, Nakagami- $m$ fading, social comparison, stochastic geometry.

\section{INTRODUCTION}

Fifth generation $(5 \mathrm{G})$ wireless networks are expected to support significantly higher capacity (1000 fold) than existing $4 \mathrm{G}$ networks. To realize this potential, major breakthroughs are required in many technical areas including, spectral efficiency, network densification, and spectrum extension [1]. In terms of spectral efficiency, several advances have already been made, including massive multiple-input multiple-output (MIMO), coordinated multi-point transmission and reception (CoMP), device-to-device (D2D) communications, cooperative communications and interference management. Network densification is achieved by off-loading cellular traffic to small cells or D2D networks, resulting in enhanced network capacity and coverage, while spectrum extension efficiently uses higher spectrum bands by means of carrier aggregation and through the use of emerging ultra short wave length technologies (e.g. millimeter-wave communications). D2D communications

This work was supported by the Engineering and Physical Sciences Research Council (EPSRC) under Grant References EP/H044191/1 and EP/L026074/1. especially are recognized as a key technology component of the $5 \mathrm{G}$ networks which will help to improve spectral efficiency and network densification simultaneously [2].

D2D communications assist direct transmission between user equipment (UE) pairs, reducing unnecessary routing of traffic to base stations (BSs) lowering the transmit power and hence interference levels. D2D UEs can simultaneously transmit with cellular UEs over the licensed band by using underlay spectrum access schemes and achieve significantly higher capacity gains than traditional cellular networks [3]. Nonetheless, this co-channel transmission results in crossmode interference between cellular and D2D UEs. In order to guarantee a quality of service (QoS) to the cellular links, underlaid spectrum access scheme needs to restrict the transmit power of the D2D nodes. However this restriction leads to a poor link quality and limited transmission rate over the D2D mode. One viable option to improve the D2D link quality is incorporating cooperative relays into D2D networks, known as relay assisted $D 2 D$ networks. By using relays, relay assisted D2D networks can virtually reduce the length of each links and achieve a higher rate than conventional D2D networks [4]-[7].

While relay assisted D2D networks offer many advantages, they also come with numerous challenges that include the difficulties in accurately modeling random node locations and evaluating the aggregative interference induced by the cellular UEs, D2D UEs, and relay nodes. Recently, stochastic geometry has received considerable attention as a useful mathematical tool for interference modeling. Specifically, stochastic geometry treats the locations of the interferer as points distributed according to a spatial point process [8]. Such an approach captures the topological randomness in the network geometry, allows high analytical flexibility and achieves an accurate performance evaluation. A common assumption in most of the related works is that the interfering nodes are distributed according to a homogeneous Poisson point process (PPP). In [9], the authors have compared two D2D spectrum sharing schemes (overlay and underlay) and evaluated the average achievable rate for each scheme based for PPP distributed UEs. In [10], the authors extended the work conducted in [9] by considering a D2D link whose length depends on the 
user density. In [11], the authors proposed a distributed relay selection scheme to minimize the total transmit power of relay assisted D2D networks.

While previous works have made significant advances from an analytical point of view, they failed to reflect two practical aspects of relay assisted D2D networks. Firstly, they commonly assumed Rayleigh distributed small scale fading. Due to its analytical simplicity, Rayleigh fading has been widely adopted in stochastic geometric analysis and achieved tractable results. However Rayleigh fading can only represent a particular category of the fading environments, which is homogeneous, linear, and circularly symmetric. If the fading environment has a dominant Line-of-sight (LOS) component or the transmission scheme includes certain type of diversity combining, then Rayleigh distribution can not be applied as the appropriate small scale fading model.

Secondly, previous works assume that cooperation is spontaneously available on demand: in other words, relay nodes are considered to unquestionably donate their resources for the good of others. However without some intervention, the rational individual strategy is defection [12]-[15]. Centralized control is one way in which this can be resolved, but this is a complex issue given that some control rests with the device owner who may have personal priorities for resource usage (e.g., battery life preservation) that conflict with those of the network.

Therefore it is necessary to consider models of cooperation that incentivise user participation. The particular form of cooperation relevant to D2D communication is indirect reciprocity [16] where individuals are required to donate resources without reciprocation from the receiving party. This is an established problem in biological and life sciences - in particular indirect reciprocity is a naturally sustained and defining feature of human populations. A universal characteristic governing such systems is the cost to benefit ratio, which captures the cost to the individual of donation in comparison to the benefit enjoyed by the recipient. When this is low the prospects for emergence of cooperation naturally increase.

Considerable research has been undertaken to establish the conditions where indirect reciprocity is sustained, which have generally used reputation as the currency through which individuals become motivated to engage in socially beneficial activities [15], [17]. In this work we adopt a fundamental underlying model for the evolution of indirect reciprocity [18] that is based on social comparison [19], where individual entities compare the reputation of each other and use this to inform their donation behaviour. This has been found embedded in a range of explanations for indirect reciprocity and therefore it is suitable to be considered for the emergence of cooperation in D2D scenarios.

Taking into account the evolution of cooperation, we consider a more realistic relay assisted D2D network where each relay node has an associated cooperation probability that evolves over time and the channel coefficients are distributed according to Nakagami- $m$ fading. The spatial locations of the D2D UEs, cellular base stations (BSs), and relay nodes are

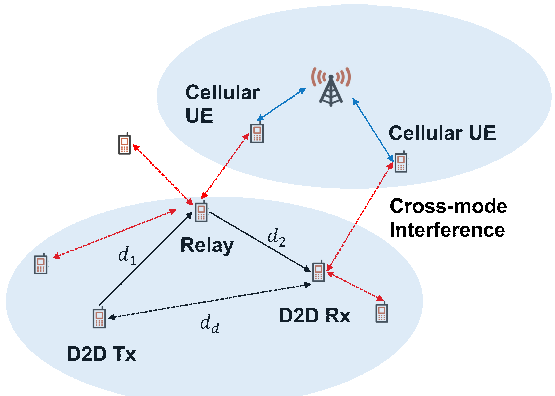

Fig. 1: System Model for Relay Network

modeled as PPP. We evaluate the end-to-end transmission rate of relay assisted D2D networks for a given probability of cooperation, and compare the effects of the evolution of the probability of cooperation using the model developed in [18].

The main contributions of this paper may be summarized as follows.

1) We analyze the outage probability and capacity of relay assisted D2D networks over Nakagami- $m$ fading using stochastic geometry.

2) We model relay cooperation as donation game based on social comparison and characterized cooperation probability in an evolutionary context. Based on the proposed evolutionary cooperation model, we compare the capacity of relay assisted D2D network over various scenarios.

3) We optimize the capacity of relay assisted D2D networks and determine the optimal mode (single- or two-hop) for a given node intensity, internode distance, and channel environment based on numerical evaluation.

The remainder of this paper is organized as follows. In Section II, we describe the system model and the fading models that will be used in this study. In Section III, we model relay cooperation probability by using the social comparison model in the context of a donation game based on social comparison. Based on this model, we evaluate the outage probability and transmission capacity of relay assisted D2D networks. We present numerical results in Section IV and conclude the paper in Section V.

\section{System And Channel Model}

As illustrated in Fig. 11, we consider a D2D network underlaid to a cellular uplink where D2D UEs access the licensed band with the cellular UEs. The cellular UEs, D2D UEs, and relay nodes are independently distributed as a marked PPP $\Phi_{c}$ with intensity $\lambda_{c}, \Phi_{d}$ with intensity $\lambda_{d}$, and $\Phi_{r}$ with intensity $\lambda_{r}$, respectively. Each marked PPP is given by

$$
\Phi_{j}=\left\{X_{j i}, d_{j i}, h_{j i}\right\}, \quad j \in \Phi=\{c, d, r\},
$$

where the $j$ subscript indicates the UE class (cellular UE, D2D UE, and relay nodes), $X_{j i}$ denotes both the node and the coordinates of the $i$-th UE in class $j, d_{j i}$ and $h_{j i}$ represents the length of the link and the fading coefficient between the $i$-th 
$\mathrm{UE}$ and the receiver, respectively. The typical D2D receiver is located at the origin and associated to the D2D UE $X_{d 0}$.

The received power from the $i$-th UE in class $j$ is $W=$ $P_{j} h_{j i} d_{j i}^{-\alpha}$, where $P_{j}$ and $\alpha>2$ denote the transmit power and the path-loss exponent, respectively. Without loss of generality, we focus on an interference-limited environment and the received SIR (Signal-to-interference ratio) at the origin is given by

$$
\mathrm{SIR}=\frac{P_{d} h_{d 0} d_{d 0}^{-\alpha}}{\sum_{j \in\{c, d\}} \sum_{i \in \Phi_{j} \backslash\left\{X_{d 0}\right\}} P_{j} h_{j i} d_{j i}^{-\alpha}} .
$$

We adopt the communication protocol proposed in [20] for relay assisted D2D networks. Initially, the D2D receiver search for a relay that is located within the relay search range. If there are closely located relay nodes, two-hop D2D transmission is utilized. Otherwise, single-hop D2D transmission is selected. Given two-hop D2D transmission, a D2D UE transmits its packet to the receiver UE during the first time slot and closely located relay nodes overhear this packet. If the received SIR at the $i$-th relay is larger than a predefined SIR threshold $T$, the $i$-th relay node becomes a potential relay and the D2D receiver chooses the best relay from the potential relay nodes set. The selected relay node uses decode and forward cooperation scheme and sends the original source packet to the D2D receiver during the second time slot. The transmission protocol is described in more detail in Section III.

Nakagami- $m$ fading is used as the small scale fading model; Nakagami- $m$ fading is a versatile model that includes Rayleigh fading and One-sided Gaussian fading as a special cases. Furthermore, it can also be used to approximate Rician fading with high accuracy. For a Nakagami- $m$ distributed channel envelope $R$, the channel coefficient $h=|R|^{2}$ in $(1)$ follows a Gamma distribution. In this case, the PDF, complementary CDF, and $j$-th moment of $h$ are respectively given by [21]

$$
\begin{array}{r}
f_{h}(x)=\frac{m^{m} x^{m-1}}{\Gamma(m)} \mathrm{e}^{-m x}, \quad \mathbb{E}\left[h^{j}\right]=\frac{\Gamma(m+j)}{\Gamma(m)}, \\
\mathbb{P}(h \geq x)=\frac{\Gamma(m, m x)}{\Gamma(m)}=\sum_{n=0}^{m-1} \frac{(m x)^{n}}{n !} \mathrm{e}^{-m x},
\end{array}
$$

where we assumed a unit spread factor without loss of generality, i.e., $\Omega=\mathbb{E}\left[R^{2}\right]=1, m$ is the shape factor, $\Gamma(t)=\int_{0}^{\infty} x^{t-1} \mathrm{e}^{-x} \mathrm{~d} x$ is the Gamma function, and $\Gamma(a, b)=$ $\int_{b}^{\infty} x^{a-1} \mathrm{e}^{-x} \mathrm{~d} x$ is the upper incomplete gamma function.

\section{Evaluation of the System Measures}

In this section, we model relay cooperation as a donation game based on social comparison, characterizing the cooperation probability using the social comparison model in an evolutionary context, then evaluate the outage probability and achievable rate of relay assisted D2D networks through a stochastic geometric framework.

\section{A. Cooperation Probability based on Fundamental Evolution- ary Principles}

To incorporate the chance of cooperation being available in D2D scenarios we have implemented a fundamental evolution- ary model of cooperation [18] and determined the probability of cooperative behaviour at different stages of evolution. This is based on a generic evolutionary algorithm in which individuals reproduce proportionally to fitness values representing the cumulative payoff produced after a number of pairwise exchanges (rounds) conducted during each generation [22], [23]. We model each exchange using the donation game, which is an economic game firstly introduced in [23] and then universally adopted for the study of indirect reciprocity [17], [24]. In our scenario the roles of donor and recipient are assigned respectively to the relay and the source node that is transmitting the data. During each interaction the relay has to decide whether or not to donate resources, the results of which are captured in changes to the donating nodes' reputation, which is assumed public in the network. Evolution reproduces strategies in proportion to the total payoff that emerges from donation: a small cost $c$ is incurred by the donor and a larger benefit $b$ is enjoyed by the recipient. We here assumed a standard value of 0.5 for the cost to benefit ratio $c / b$.

Donation decisions are governed by the strategy of an individual node, each strategy defined by an action and an assessment rule [24]. We propose a novel set of action rules [18] based on social comparison principles defined as follows. Given a donor $i$ and and recipient $j$ with reputations $s_{i}$ and $s_{j}$ respectively, donor $i$ assesses the reputation $s_{j}$ of $j$, against their own reputation, $s_{i}$, with three possible outcomes, establishing either: approximate similarity $\left(s_{j}-\Delta \leq s_{i} \leq\right.$ $\left.s_{j}+\Delta\right)$, upward self-comparison $\left(s_{j}>s_{i}+\Delta\right)$, or downward self-comparison $\left(s_{j}<s_{i}-\Delta\right)$. A node's strategy allows donation in response to any combination of these possible comparisons. Updating reputation in response to donation decisions is known as the assessment rule. This is a further variable that affects evolution [18], because reputation informs decision making. We adopt a variation of the original standing assessment approach [22], [24], which justifies participant defection in circumstances where they are of a lower standing than themselves. This is defined by incrementing the donor $i$ reputation after each donation made while decrementing it only when a defection occurs in light of a request from a recipient $j$ with at least the same reputation value of the donor's [18]. This framework provides an environment for cooperation to evolve under wide-ranging $c / b$ ratios [22], [23]. Similarly to these works we have bounded the reputation values within the finite range \pm 5 .

The distribution probability of cooperation of the network nodes at different stages (generations) of the evolutionary simulation is visualized by the histograms in Fig. 2, as empirically retrieved from a number of simulation runs with different random seeds. Here the abscissa represents the 'probability $\xi_{i}$ that a node $i$ cooperates with a randomly chosen node in a randomly chosen round at a given generation'.

At the beginning of the simulation all nodes act according to randomly assigned strategies, including full cooperation and defection. After about one hundred generations (in the example shown but often requiring less, in the order of tenth, and even units for more favourable $c / b$ ratios) the network converges 


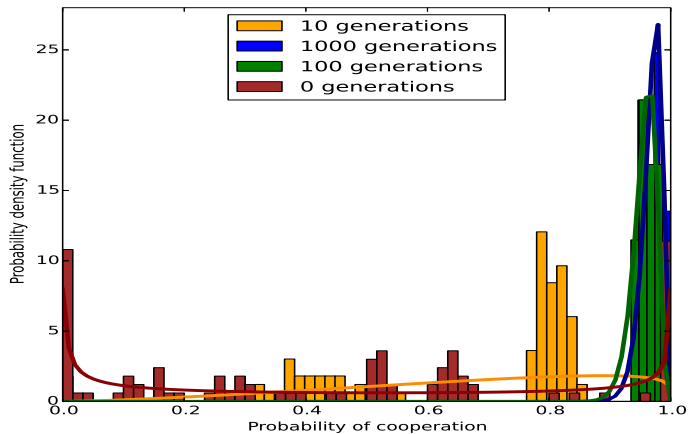

Fig. 2: Distributions of the probability of cooperation produced by our experimentation at different generations of the evolutionary simulation.

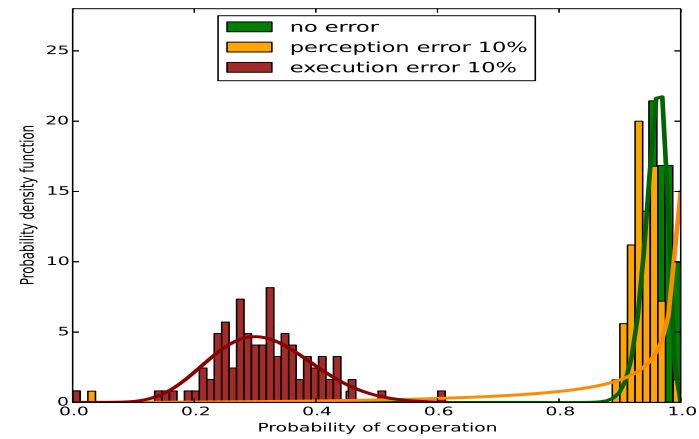

Fig. 3: Distributions of the probability of cooperation produced by our experimentation in presence of error at generation 100 of the evolutionary simulation.

to a configuration with all nodes adopting a dominant strategy of 'upward or similar comparison', i.e., 'donating in light of a request from nodes of higher or similar reputation while defecting otherwise'. This has been identified in [18] as an important strategy when donation decisions are based on selfcomparison, as first considered in [12].

In order to apply this to the wireless relay D2D scenario we approximate these distributions of cooperation with a Beta distribution fitting (solid lines in Fig. 2), in which we approximate the gamma function characterizing this distribution by applying moment matching [25].

The probability of cooperation worsens when different types and percentages of errors are introduced. In particular two types of errors have been considered: execution errors in the implementation of the actual actions performed by the nodes (donors) representing, for example, dropped connections due to interference; and perception errors in the representation of other nodes' image, while the consequent actions are assumed to be performed correctly [17], [24].

Fig. 3 shows the distribution of the probability of cooperation at generation 100 of the evolutionary simulation for the two different types of error. With errors in the perception of reputation, cooperation is achieved and sustained after a maximum of 100 generations, as in the case without any error, whereas for errors in execution we need more generations (1000 in the example shown but often requiring less) to converge to high cooperation levels (here we have considered $10 \%$ rates).

During the generations before convergence, configurations of low cooperation can appear in the network. Nevertheless, these states remain in place only for a limited number of generations, then the system is able to return to the dominant strategy of devices donating in response to recipients having 'upward or similar comparison of reputation' that produces a configuration close to the ideal case of $100 \%$ cooperation.

\section{B. Outage Probability and Capacity}

1) Single-Hop D2D: A successful transmission occurs when the received SIR in (2) is larger than a predefined SIR threshold $T$. Then, the probability of successful transmission over a single-hop D2D is defined as follows

$$
\begin{aligned}
\mathbb{P}_{s}^{1 \text {-hop }} & \triangleq \mathbb{P}(\operatorname{SIR}>T)=\mathbb{P}\left(h_{d 0}>d_{d 0}^{\alpha} T I\right) \\
& =\mathbb{E}_{I}\left[\sum_{n=0}^{m-1} \frac{\left(m d_{d 0}^{\alpha} T I\right)^{n}}{n !} \mathrm{e}^{-m d_{d 0}^{\alpha} T I}\right],
\end{aligned}
$$

where $I=\sum_{j \in\{c, d\}} \sum_{i \in \Phi_{j} \backslash\left\{X_{d 0}\right\}}\left(P_{j} / P_{d}\right) h_{j i} d_{j i}^{-\alpha}$, 22 is applied in the first equality, and (3) is utilized in the second equality. The expectation in (4) can be evaluated by using the high order derivatives of the Laplace transform of $I$ as follows

$$
\mathbb{E}_{I}\left[\left(c_{0} I\right)^{n} \exp \left(-s c_{0} I\right)\right]=(-1)^{n} \frac{\partial^{n} \mathcal{L}_{I}(s)}{\partial s^{n}},
$$

where $c_{0}=m d_{d 0}^{\alpha} T, \delta=\frac{2}{\alpha}, \mathcal{L}_{I}(s)$ is derived as

$$
\begin{aligned}
\mathcal{L}_{I}(s) & =\mathbb{E}_{\Phi_{j}, h}\left[\mathrm{e}^{-s c_{0} I}\right] \\
& =\mathbb{E}\left[\mathrm{e}^{\left.-s c_{0}\left\{\sum_{i \in \Phi_{c}} \frac{P_{c}}{P_{d}} h_{c i} d_{c i}^{-\alpha}+\sum_{i \in \Phi_{d}} h_{d i} d_{d i}^{-\alpha}\right\}\right]}\right. \\
& =\exp \left(-c_{\alpha} T^{\delta} d_{d 0}^{2} s^{\delta}\left(\lambda_{d}+\lambda_{c}\left(\frac{P_{c}}{P_{d}}\right)^{\delta}\right)\right),
\end{aligned}
$$

by applying the well-known probability generating functional (PGFL) of a PPP [8] in the last equality and the constant $c_{\alpha}$ is determined using the channel statistics in (3) as follows

$$
c_{\alpha} \triangleq \pi m^{\delta} \Gamma(1-\delta) \mathbb{E}\left[h_{j i}^{\delta}\right]=\frac{\pi m^{\delta} \Gamma(1-\delta) \Gamma(m+\delta)}{\Gamma(m)} .
$$

Hence, the outage probability and transmission capacity of a single-hop D2D over Nakagami- $m$ fading are given by [20]

$$
\begin{aligned}
\mathbb{P}_{o}^{1-\text { hop }} & \triangleq \mathbb{P}(\mathrm{SIR} \leq T)=1-\mathbb{P}_{s}^{1-\text {-hop }} \\
& =1-\left.\sum_{n=0}^{m-1} \frac{(-1)^{n}}{n !} \frac{\partial^{n} \mathcal{L}_{I}(s)}{\partial s^{n}}\right|_{s=1}, \\
C^{1 \text {-hop }} & \triangleq \lambda_{d} \log (1+T) \mathbb{P}_{s}^{1-\text { hop }} \\
& =\left.\lambda_{d} \log (1+T) \sum_{n=0}^{m-1} \frac{(-1)^{n}}{n !} \frac{\partial^{n} \mathcal{L}_{I}(s)}{\partial s^{n}}\right|_{s=1} .
\end{aligned}
$$


2) Two-Hop D2D: We denote $d_{k}$ as the link length of the $k$-th hop, $d_{d}$ as the internode distance between source and destination, and $r$ as the distance from the relay to the midpoint between source and destination. The transmitter communicates directly with the receiver in a single-hop D2D, whereas for two-hop D2D, the link between transmitter and receiver is assumed to be unreliable and the transmission occurs only through the relay.

Assuming equal transmit power for the source and relay nodes, the probability of successful transmission over two-hop D2D is defined as follows

$$
\begin{aligned}
\mathbb{P}_{s}^{2-\text { hop }} & \triangleq \mathbb{P}\left(\mathrm{SIR}_{1}>T\right) \mathbb{P}\left(\mathrm{SIR}_{2}>T\right) \\
& =\left.\sum_{n=0}^{m-1} \frac{(-1)^{n}}{n !} \frac{\partial^{n}}{\partial s^{n}} \mathcal{L}_{I_{1}}(s) \mathcal{L}_{I_{2}}(s)\right|_{s=1},
\end{aligned}
$$

where $c_{\alpha}$ is given in (7), $\delta=\frac{2}{\alpha}$, and

$$
\begin{aligned}
\mathcal{L}_{I_{1}}(s) \mathcal{L}_{I_{2}}(s) & =\mathrm{e}^{-c_{\alpha}(s T)^{\delta}\left(d_{1}^{2}+d_{2}^{2}\right)\left(\lambda_{d}+\lambda_{c}\left(\frac{P_{c}}{P_{d}}\right)^{\delta}\right)} \\
& =\mathrm{e}^{-c_{\alpha}(s T)^{\delta}\left(\frac{d_{d}^{2}}{2}+2 r^{2}\right)\left(\lambda_{d}+\lambda_{c}\left(\frac{P_{c}}{P_{d}}\right)^{\delta},\right)},
\end{aligned}
$$

by using cosine rule, i.e., $\frac{d_{d}^{2}}{2}+2 r^{2}=d_{1}^{2}+d_{2}^{2}$. Similarly, the transmission capacity of two-hop D2D is given by

$$
\begin{aligned}
& C^{2 \text {-hop }}(r) \triangleq \frac{\lambda_{d}}{2} \log (1+T) \mathbb{P}_{s}^{2-\text { hop }} \\
& =\left.\frac{\lambda_{d}}{2} \log (1+T) \sum_{n=0}^{m-1} \frac{(-1)^{n}}{n !} \frac{\partial^{n}}{\partial s^{n}} \mathcal{L}_{I_{1}}(s) \mathcal{L}_{I_{2}}(s)\right|_{s=1},
\end{aligned}
$$

which is a function of the relay location $r$.

We adopt a relay selection scheme similar to [20] where the authors choose the relay closest to the midpoint between the transmitter and the receiver. In contrast, we introduce a Beta distributed random variable $\xi_{i}$ that indicates the cooperation probability of each relay node. Then, we choose the relay that maximizes $\xi_{i}\left\|X_{r i}\right\|^{-\alpha}$ (or minimizes $\xi_{i}^{-1 / \alpha}\left\|X_{r i}\right\|$ ), $i \in \Phi_{r}$. Conceptually, the cooperation probability is interpreted as a random fluctuation around each relay location $X_{r, i} \in \Phi_{r}$ and the combined effect of relay node location and cooperation probability are incorporated into the relay selection policy. Due to displacement theorem [26], the transformed point process $Y_{r, i}=\xi_{i}^{-\frac{1}{\alpha}} X_{r, i}$ is also a PPP with density $\lambda_{\xi}=\lambda_{r} \mathbb{E}\left[\xi^{\delta}\right]$. Note that the $\delta$-th order moment of Beta distributed random variable is $\mathbb{E}\left[\xi^{\delta}\right]=B(\alpha+\delta, \beta) / B(\alpha, \beta)$ for a given shape parameters $\alpha$ and $\beta$. Then, the $\mathrm{CDF}$ and $\mathrm{PDF}$ of $Y_{r, i}$ are respectively given by

$$
\begin{aligned}
\mathbb{P}\left(\left\|Y_{r i}\right\|>r\right) & =\mathrm{e}^{-\pi r^{2} \lambda_{r} \mathbb{E}\left[\xi^{\delta}\right]}, \\
f_{\left\|Y_{r i}\right\|}(r) & =2 \pi r \lambda_{r} \mathbb{E}\left[\xi^{\delta}\right] \mathrm{e}^{-\pi r^{2} \lambda_{r} \mathbb{E}\left[\xi^{\delta}\right]},
\end{aligned}
$$

where $P_{\mathcal{N}}(r) \triangleq \mathbb{P}\left(\left\|Y_{r i}\right\|>r\right)$ is the null probability to find a relay within a search range $\left\|Y_{r i}\right\| \leq r$.

Two-hop D2D transmission is utilized if there is a relay within the relay search range. Otherwise, single-hop D2D transmission is selected. Hence, the average transmission capacity of relay assisted D2D is evaluated as

$$
\begin{aligned}
C^{\text {Relay }} & =\left(1-P_{\mathcal{N}}(R)\right) \int_{0}^{R} C^{2 \text {-hop }}(r) f_{\left\|Y_{r i}\right\|}(r) \mathrm{d} r \\
& +P_{\mathcal{N}}(R) C^{1 \text {-hop }}
\end{aligned}
$$

where $R$ is the relay search range that needs to be optimized,

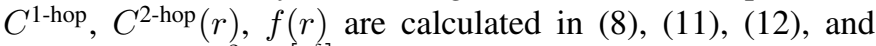
$P_{\mathcal{N}}(R)=\mathrm{e}^{-\pi R^{2} \lambda_{r} \mathbb{E}\left[\xi^{\delta}\right]}$.

Remark 1. The $n$-th order derivatives in (8) and (11) can be numerically evaluated by using Faa di Bruno's formula as follows [27]

$$
\frac{\partial^{n}}{\partial x^{n}} f(g(x))=f(g(x)) B_{n}\left(g^{(1)}(x), \cdots, g^{(n)}(x)\right),
$$

where $B_{n}\left(x_{1}, \cdots, x_{n}\right)$ is the $n$-th complete Bell polynomial.

\section{NUMERICAL RESULTS}

In this section, we numerically evaluate and optimize the capacity of a relay assisted D2D network to guarantee QoS to the cellular UEs. All of the simulations were carried out using the following parameters: cellular UE intensity $\lambda_{c}=10^{-5}$, SIR threshold $T=3$, path-loss exponent $\alpha=4$, and D2D pair internode distance $d_{d}=10$.

For optimization, we consider a two-dimensional grid composed of D2D UEs intensity $\lambda_{d}$ and relay node intensity $\lambda_{r}$. At each grid point, we numerically perform the following optimization strategy

$$
\underset{R, P_{c} / P_{d}}{\arg \max } C^{\text {Relay }} \text { subject to } \mathbb{P}_{o}^{\text {Cellular }} \leq \theta_{c},
$$

where we assumed $\theta_{c}=0.05, d_{c}=10$, and the outage probability of a cellular link $\mathbb{P}_{o}^{\text {Cellular }}$ is evaluated by 8 , with the following Laplace transform $\mathcal{L}_{I_{c}}(s)$

$$
\mathcal{L}_{I_{c}}(s)=\exp \left(-c_{\alpha} T^{\delta} d_{c}^{2} s^{\delta}\left(\lambda_{c}+\lambda_{d}\left(\frac{P_{d}}{P_{c}}\right)^{\delta}\right)\right) .
$$

Fig. 4 compares the capacity of a single-hop D2D to that of a two-hop D2D network for different Nakagami- $m$ shape factors. We fixed the relay node intensity $\lambda_{r}=10^{-2}$ and assumed $\xi_{i}=1$ for every relay nodes to determine the theoretical performance bound. As illustrated in Fig. 4, relay assisted D2D transmission achieves a higher rate than the single hop D2D, whereas a rich scattering environment, i.e., large $m$, decreases the transmission capacity. We note that the capacity increases for a small UE intensity $\lambda_{d}$, then decreases after a certain threshold. This effect is due to the fact that every node is transmitting at a same power; in a dense network, the interference increases as $\lambda_{d}$ increases, decreasing the received SIR and the overall network performance.

Fig. 5 compares the capacity of a two-hop D2D network for different distributions of the cooperation probability $\xi_{i}$. The curves for single-hop and two-hop with $100 \%$ cooperation corresponds to the case of $\xi_{i}=0$ and $\xi_{i}=1$, respectively. For the two blue curves in the middle, we modeled relay 


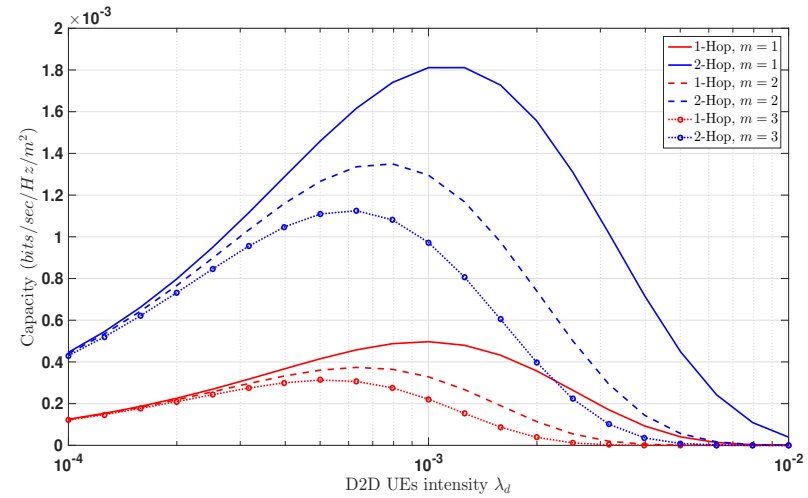

Fig. 4: Capacity versus D2D UE intensity $\lambda_{d}$ for different Nakagami shape factor $m$, relay node intensity $\lambda_{r}=10^{-2}$, cooperation probability $\xi_{i}=1$.

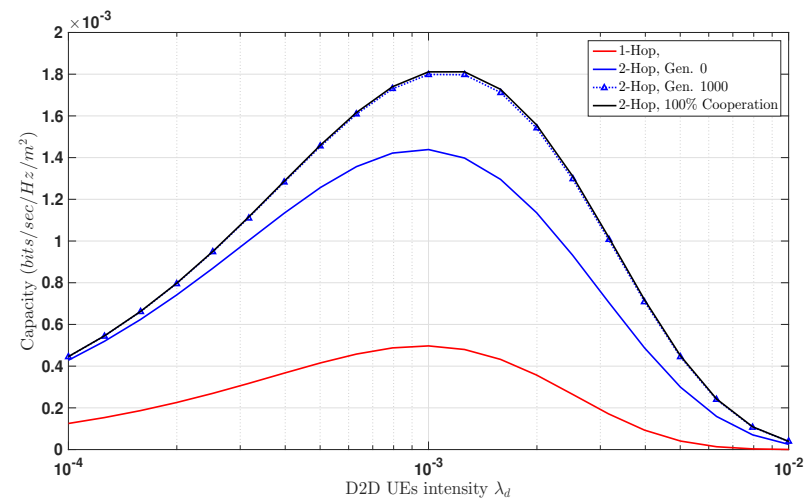

Fig. 5: Capacity versus D2D UEs intensity $\lambda_{d}$ for different cooperation probability $\xi_{i}$, relay node intensity $\lambda_{r}=10^{-2}$, Nakagami shape factor $m=1$.

cooperation as a donation game based on social comparison and evolved the distribution of cooperation over generations. The solid blue curve corresponds to the generation 0 case where each relay randomly chooses whether to cooperate or not without any side information. The dashed blue curve corresponds to generation 100 and it approaches the ideal case of $100 \%$ cooperation which is the theoretical bound. This result indicates that after a sufficient number of generations, they all converge to a configuration in which cooperation is sustained in the population (and all nodes adopt the same dominant cooperative strategy based on social comparison) without the need to enforce any external mechanisms.

Figures 6 and 7 show the same results when errors are considered in the reputation model based on social comparison. While perception errors only marginally degrade the overall performance, the introduction of errors in the execution requires a longer number of generations to sustain high cooperation levels. Note that, in earlier stages (gen. 100) the network can in this case temporarily present intermediate configurations of low cooperation that could drop the capacity below the initial values obtained assuming a random distribution of strategies (gen. 0). However, these low

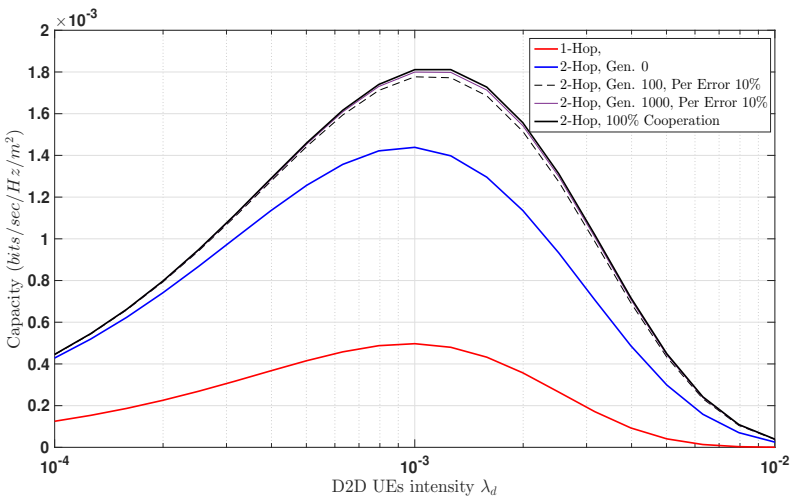

Fig. 6: Capacity versus D2D UEs intensity $\lambda_{d}$ for different number of generations with Perception error $10 \%, \lambda_{r}=10^{-2}$, and Nakagami shape factor $m=1$.

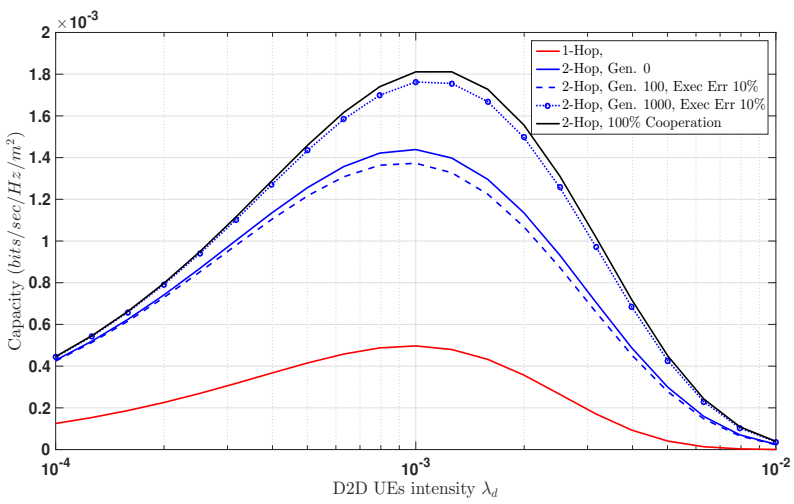

Fig. 7: Capacity versus D2D UEs intensity $\lambda_{d}$ for different number of generations with Execution error $10 \%, \lambda_{r}=10^{-2}$, and Nakagami shape factor $m=1$.

cooperation states are not stable and the system nodes are able to promptly recover towards the dominant strategy until this final configuration eventually stabilises the performance towards high capacity levels, still remaining close to the ideal case of $100 \%$ cooperation.

In Fig. 8 and Fig. 9, we evaluated the ratio between the capacity of relayed D2D to that of single-hop D2D $\nu=$ $C^{\text {Relay }} / C^{1 \text {-hop }}$ on each grid point $\left(\lambda_{d}, \lambda_{r}\right)$. We assumed $\xi_{i}=1$ for every relay nodes and considered Nakagami distribution with $m=1$ in Fig. 8 and $m=3$ in Fig. 9 To normalize the ratio $\nu \in[1, \infty)$ into a unit range $[0,1]$, we defined $\eta$ parameter as $\eta=\log (\nu) /(\log (\nu)+1)$, where $\eta=0$ for $C^{\text {Relay }}=C^{1 \text {-hop }}$ and $\eta \rightarrow 1$ as $\nu \rightarrow \infty$. The dark blue region corresponds to $\eta=0$ that provides no relaying gain, i.e., it is optimal to use single-hop D2D in the dark blue region. As the operational range moves towards the brighter colors in north-east direction, relay assisted D2D communications achieves significantly larger transmission capacities than the single-hop D2D. Hence, in bright color region (yellow or green), it is optimal to use two-hop D2D; relay assisted D2D provides a capacity gain only in a dense network with large $\lambda_{d}$ and $\lambda_{r}$. We also note that the dark blue region in Fig. 9 


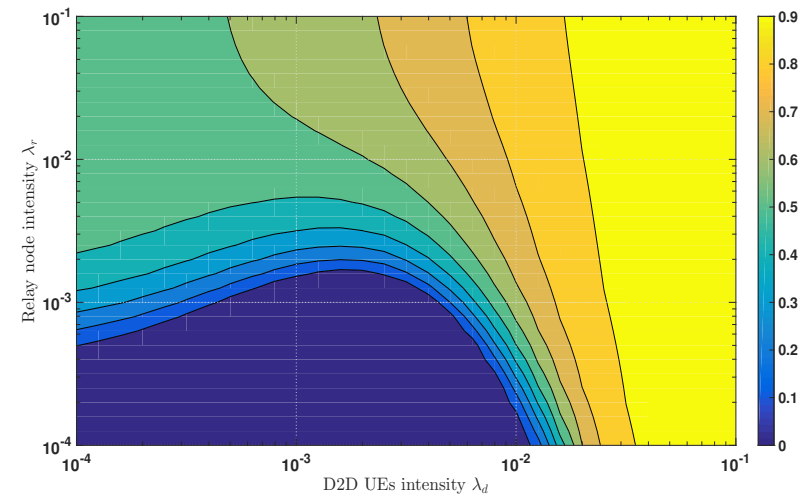

Fig. 8: Normalized capacity ratio $C^{\text {Relay }} / C^{1 \text {-hop }}$ for cooperation probability $\xi_{i}=1$ and Nakagami shape factor $m=1$.

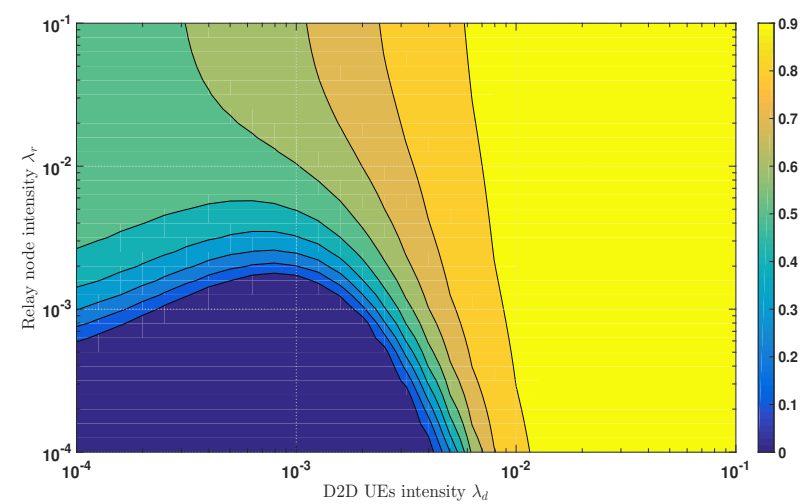

Fig. 9: Normalized capacity ratio $C^{\text {Relay }} / C^{1-h o p}$ for cooperation probability $\xi_{i}=1$ and Nakagami shape factor $m=3$.

$(m=3)$ is smaller than that in Fig. $8(m=1)$. This indicates that the benefit of using relay assisted D2D stands out in a rich scattering environment (large $m$ ) and the optimal mode selection between single-hop D2D and two-hop D2D should be determined based on the given channel condition.

\section{CONCLUSION}

In this paper, we have considered a relay assisted D2D network underlaid to an uplink cellular network, where the spatial locations of the nodes are modeled as PPP. We proposed a social comparison model in an evolutionary context to characterize relay cooperation probability. Using the proposed comparison model with stochastic geometric framework, we evaluated the outage and capacity of relay assisted D2D network. Specifically, we observed that after a sufficient number of generations, the cooperation probability follows the natural rules of evolution and all relay nodes adopt the same dominant cooperative strategy based on social comparison without the need to enforce any external mechanisms. Also, we observed that the benefit of relaying stands out in a dense network with rich scattering channel. Finally, we provided numerical results to demonstrate the performance gains of relay assisted D2D networks compared to single hop D2D networks.

\section{REFERENCES}

[1] H. Ishii, Y. Kishiyama, and H. Takahashi, "A Novel Architecture for LTE-B:C-plane/U-plane Split and Phantom Cell Concept," in 2012 IEEE GLOBECOM, Anaheim, USA, Dec. 2012.

[2] M. Tehrani, M. Uysal, and H. Yanikomeroglu, "Device-to-Device Communication in 5G Cellular Networks: Challenges, Solutions, and Future Directions," IEEE Commun. Mag., vol. 52, no. 5, pp. 86-92, May 2014.

[3] A. Asadi, Q. Wang, and V. Mancuso, "A Survey on Device-to-Device Communication in Cellular Networks," IEEE Commun. Surv. Tutorials, vol. PP, no. 99, pp. 1-1, Jan. 2014.

[4] T. Cover and A. E. Gamal, "Capacity theorems for the relay channel," IEEE Trans. Inf. Theory, vol. 25, no. 5, pp. 572-584, 1979.

[5] A. Sendonaris, E. Erkip, and B. Aazhang, "User cooperation diversity Part I: System description," IEEE Trans. Commun., vol. 51, no. 11, pp. 1927-1938, 2003.

[6] J. N. Laneman, D. N. C. Tse, and G. W. Wornell, "Cooperative diversity in wireless networks: Efficient protocols and outage behavior," IEEE Trans. Inf. Theory, vol. 50, no. 12, pp. 3062-3080, 2004.

[7] G. Kramer, M. Gastpar, and P. Gupta, "Cooperative strategies and capacity theorems for relay networks," IEEE Trans. Inf. Theory, vol. 51, no. 9, pp. 3037-3063, 2005.

[8] M. Haenggi, Stochastic geometry for wireless networks. Cambridge University Press, 2013, vol. I.

[9] X. Lin, J. G. Andrews, and A. Ghosh, "Spectrum Sharing for Deviceto-Device Communication in Cellular Networks," IEEE Trans. Wirel. Commun., vol. 13, no. 12, pp. 1-31, Dec. 2014.

[10] G. George, R. K. Mungara, and A. Lozano, "An Analytical Framework for Device-to-Device Communication in Cellular Networks," IEEE Trans. Wirel. Commun., p. 30, 2014.

[11] X. Ma, R. Yin, G. Yu, and Z. Zhang, "A distributed relay selection, method for relay assisted Device-to-Device communication system," IEEE Int. Symp. Pers. Indoor Mob. Radio Commun. PIMRC, pp. 10201024, 2012.

[12] S. M. Allen, G. Colombo, and R. M. Whitaker, "Cooperation through self-similar social networks," ACM Transactions on Autonomous and Adaptive Systems (TAAS), vol. 5, no. 1, p. 4, 2010.

[13] — "Forming social networks of trust to incentivize cooperation," in System Sciences, 2009. HICSS'09. 42nd Hawaii International Conference on. IEEE, 2009, pp. 1-10.

[14] R. Axelrod and W. D. Hamilton, "The evolution of cooperation," Science, vol. 211, no. 4489, pp. 1390-1396, 1981.

[15] M. A. Nowak, "Five rules for the evolution of cooperation," science, vol. 314, no. 5805, pp. 1560-1563, 2006.

[16] R. D. Alexander, The biology of moral systems. Transaction Pub., 1987.

[17] H. Brandt, H. Ohtsuki, Y. Iwasa, and K. Sigmund, "A survey of indirect reciprocity," in Math. for Eco. and Env. Sci. Springer, 2007, pp. 21-49.

[18] R. M. Whitaker, G. Colombo, S. M. Allen, and R. I. M. Dunbar, "A dominant social comparison heuristic unites alternative mechanisms for the evolution of indirect reciprocity," Submitted, 2016.

[19] A. P. Buunk and F. X. Gibbons, "Social comparison: The end of a theory and the emergence of a field," Organizational Behavior and Human Decision Processes, vol. 102, no. 1, pp. 3-21, 2007.

[20] Z. Lin, Y. Li, S. Wen, Y. Gao, X. Zhang, and D. Yang, "Stochastic geometry analysis of achievable transmission capacity for relay-assisted Device-to-Device networks," in Int. Conf. Comm., 2014, pp. 2251-2256.

[21] M. Salehi and J. Proakis, Digital Communications. McGraw-Hill Higher Education, 2007, vol. 2007.

[22] O. Leimar and P. Hammerstein, "Evolution of cooperation through indirect reciprocity," Proceedings of the Royal Society of London B: Biological Sciences, vol. 268, no. 1468, pp. 745-753, 2001.

[23] M. A. Nowak and K. Sigmund, "Evolution of indirect reciprocity by image scoring," Nature, vol. 393, no. 6685, pp. 573-577, 1998.

[24] - "Evolution of indirect reciprocity," Nature, vol. 437, no. 7063, pp. 1291-1298, 2005

[25] R. W. Heath, M. Kountouris, and T. Bai, "Modeling heterogeneous network interference using poisson point processes," IEEE Trans. Signal Process., vol. 61, no. 16, pp. 4114-4126, 2013.

[26] H. S. Dhillon and J. G. Andrews, "Downlink rate distribution in heterogeneous cellular networks under generalized cell selection," IEEE Wirel. Commun. Lett., vol. 3, no. 1, pp. 42-45, 2014.

[27] H. N. Huang, S. A. M. Marcantognini, and N. J. Young, "Chain rules for higher derivatives," Math. Intell., vol. 28, no. 2, pp. 61-69, 2006. 